\title{
Sadhana polynomial and its index of hexagonal system $B_{a, b}$
}

\author{
Mohammad Reza Farahani \\ Department of Applied Mathematics, Iran University of Science and Technology (IUST), Narmak, Tehran 16844, Iran
}

\section{Email address:}

MR_Farahani@Mathdep.iust.ac.ir

\section{To cite this article:}

Mohammad Reza Farahani. Sadhana Polynomial and its Index of Hexagonal System $B_{a, b}$. International Journal of Computational and Theoretical Chemistry. Vol. 1, No. 2, 2013, pp. 7-10. doi: 10.11648/j.ijctc.20130102.11

\begin{abstract}
Let $G$ be an arbitrary graph. Two edges $e=u v$ and $f=x y$ of $G$ are called co-distant (briefly: $e$ co $f$ ) if they obey the topologically parallel edges relation. The Sadhana polynomial $S d(G, x)$, for counting $q o c$ strips in $G$ was defined by Ashrafi and co-authors as $S d(G, x)=\sum_{c} m(G, c) \mathrm{x}^{|E(G)|-c}$, where $m(G, c)$, being the number of $q o c$ strips of length $c$. This polynomial is most important in some physico chemical structures of molecules. In this paper, we compute the Sadhana polynomial and its index of an important class of benzenoid system.
\end{abstract}

Keywords: Molecular Graph, Omega Polynomial, Sadhana Polynomial, Benzenoid, Qoc Strip, Cut Method, Orthogonal Cut

\section{Introduction}

For a molecular graph $G$, there exits many topological indices in mathematical chemistry. Mathematical chemistry is a branch of theoretical chemistry for discussion and prediction of the molecular structure using mathematical methods without necessarily referring to quantum mechanics. Chemical graph theory is a branch of mathematical chemistry which applies graph [1-3].

A topological index of $G$ is a numeric quantity, derived following certain rules in Chemistry, which can be used to characterize the property of molecule and is invariant on the automorphism of the graph.

The Wiener index is the first topological index recognized in chemical graph theory, and it is often referred to as the topological index [3]. Wiener index defined as

$$
W(G)=\frac{1}{2} \sum_{u \in V(G)} \sum_{v \in V(G)} d(u, v)
$$

where the distance $d(u, v)$ between two vertices $u$ and $v$ is the number of edges in a shortest path connecting them.

Let $G$ be a simple connected graph in chemical graph theory. The vertex set and edge set of $\mathrm{G}$ denoted by $V(G)$ and $E(G)$ respectively and its vertices correspond to the atoms and the edges correspond to the bonds.

Two edges $e=u v$ and $f=x y$ of $G$ are called codistant (briefly: $e(c o f)$ if they obey the topologically parallel edges relation [4]. For some edges of a connected graph $G$ there are the following relations satisfied [5-10]

$$
\begin{gathered}
e \operatorname{co} e \\
e \operatorname{cof} \leftrightarrow f \operatorname{co} e \\
e \operatorname{cof} \& f \operatorname{coh} \rightarrow f \operatorname{coh}
\end{gathered}
$$

though the last relation is not always valid. Set $C(e):=\{f \in E(G) \mid e$ co $f\}$. If the relation "co" is transitive on $C(e)$ then $\mathrm{C}(\mathrm{e})$ is called an orthogonal cut "oc" of the graph $G$. Thus for a co-graph $G$, there is the union of disjoint orthogonal cuts for $i \neq j$ and $i, j=1,2, \ldots, k$ as $E(G)=\mathcal{C}_{1} \cup \mathcal{C}_{2} \cup \ldots \cup \mathcal{C}_{k-1} \cup \mathcal{C}_{k}$ and $\mathcal{C}_{i} \cap \mathcal{C}_{j}=\varnothing$,

The Omega Polynomial $\Omega(G, x)$ for counting qoc strips in $G$ was defined by Diudea as

$$
\Omega(G, x)=\sum_{c} m(G, c) x^{c}
$$

where $m(G, c)$, be the number of qoc strips of length $c$ (i.e., the number of cut-off edges) in the graph $G$. The summation runs up to the maximum length of qoc strips in $G$.

In 2008, Ashrafi and co-authors [11] introduced the Sadhana polynomial $S d(G, x)$, was defined as

$$
S d(\mathrm{G}, \mathrm{x})=\sum_{c} m(G, c) \mathrm{x}^{|E(G)|-c}
$$

The Sadhana index $S d(G)$, for counting qoc strips in $G$ was defined by Khadikar et.al $[12,13]$ as first derivative of 
sadhana polynomial (in $x=1)$,

$$
S d(\mathrm{G})=\sum_{c} m(G, c)(|E(G)|-c)
$$

For some chemical applications of these counting polynomial see papers [14-20]. The aim of this study is to compute the Sadhana polynomial and Sadhana index of a benzenoid system and called "hexagonal system $B_{a, b}$ ". The hexagonal system $B_{a, b}$ is family of molecular graph, which consist several copy of benzene $C_{6}$ on circumference. Shui Ling-Ling et al. defined a new hexagonal system named jagged-rectangle. An $a \times b$ hexagonal jagged-rectangle whose shape forms a rectangle and the number of hexagonal cells in each chain alternate $a$ and $a-1$. For $a \geq 2$, the vertex set of $B_{a, b}$ defined as [21-23].

$$
\begin{aligned}
& V\left(B_{a, b}\right)=\{(x, y) \mid 0 \leq x \leq 2 a, 0 \leq y \leq 2 b-1\} \\
& \cup\{(x,-1) \mid 0 \leq x \leq 2 a-1\} \cup\{(x, 2 b) \mid 1 \leq x \leq 2 a-1\}
\end{aligned}
$$

Reader can see general representation of this benzenoid system in Figure 1.
1

2
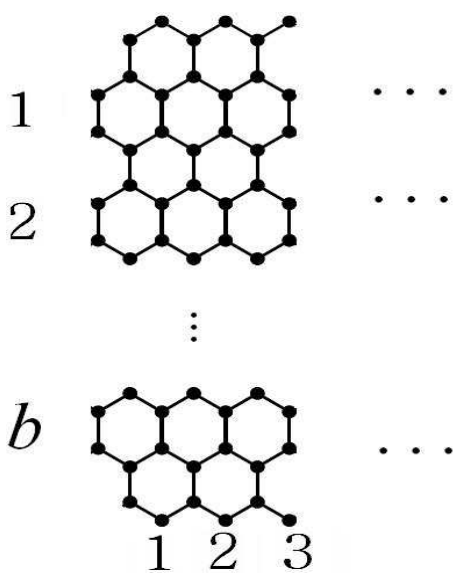

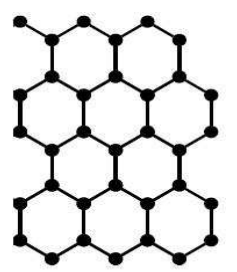

:

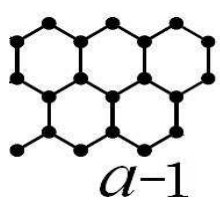

Figure. 1. A general representation of the hexagonal system $B_{a, b}(a, b \geq 1)$.

\section{Main Results}

In this section Sadhana polynomial and its index of hexagonal system $B_{a, b}$ are determined. For this computation, suppose that $G=B_{a, b}$ has $4 a b+4 a+2 b-2$ vertices and $6 a b+5 a+b-4$ edges. In the following theorem we compute the Sadhana polynomial of $G$ (Figure1).

Theorem 1: The Sadhana polynomial of hexagonal system $B_{a, b}$ is equal to

- $\quad \forall a \geq b+2, S d\left(B_{a, b}, x\right)=(b+1) x^{\left|E\left(B_{a, b}\right)\right|-a}+b x^{\left|E\left(B_{a, b}\right)\right|-a-1}$

$$
+4 \sum_{i=1}^{b} \mathrm{x}^{\left|E\left(B_{a, b}\right)\right|-2 i-1}+2(a-b-1) x^{\left|E\left(B_{a, b}\right)\right|-2 b-2}
$$

- $\quad \forall a \leq b+1, S d\left(B_{a, b}, x\right)=(b+1) x^{\left|E\left(B_{a, b}\right)\right|-a}+b x^{\left|E\left(B_{a, b}\right)\right|-a-1}$

$$
+4 \sum_{i=1}^{a-1} \mathrm{x}^{\left|E\left(B_{a, b}\right)\right|-2 i-1}+2(b-a+1) x^{\left|E\left(B_{a, b}\right)\right|-2 a}
$$

Then the Sadhana index of $B_{a, b}$ is

- $\forall a \geq b+2$,

$S d\left(B_{a, b}\right)=12 a^{2} b+24 a b^{2}+10 a^{2}+14 a b-2 b^{2}-16 a-14 b+6$

- $\forall a \leq b+1$,

$S d\left(B_{a, b}\right)=12 a^{2} b+24 a b^{2}+10 a^{2}+10 a b+4 b^{2}-18 a-18 b+8$

Proof. Let $G=B_{a, b}$ be the hexagonal system. By using the Cut Method, and by using following tables and figures the proof is easy. Cut Method and its general form studied by $S$. Klavzar [24] and used in paper series [8, 19, 20, 23-28]. Now by using this method, it is easy to see that hexagonal system is a co-graph. All distinct qoc strips of $G$ are shown in Figure 2 and numerated in Table 1 and Table 2.

Table 1. The number of co-distant edges, when $a \geq b+2$.

\begin{tabular}{llc}
\hline quasi-orthogonal cuts & Number of co-distant edges & No \\
\hline$C_{2 i+1} \forall i=0, \ldots, b$ & 1 & $a$ \\
$C_{2 i} \forall i=1, \ldots, b$ & 1 & $a+1$ \\
$\mathcal{C}_{i} \forall i=1, \ldots, b$ & 4 & $2 i+1$ \\
$\mathcal{C}_{b+1}$ & $2(a-b-1)$ & $2 b+2$ \\
\hline
\end{tabular}

Table 2. The number of co-distant edges, when $a \leq b+1$.

\begin{tabular}{lll}
\hline quasi-orthogonal cuts & Number of co-distant edges & No \\
\hline$C_{2 i+1} \forall i=0, \ldots, b$ & 1 & $a$ \\
$C_{2 i} \forall i=1, \ldots, b$ & 1 & $a+1$ \\
$\mathcal{C}_{i} \forall i=1, \ldots, a-1$ & 4 & $2 i+1$ \\
$\mathcal{C}_{a}$ & $2(b-a+1)$ & $2 a$ \\
\hline
\end{tabular}
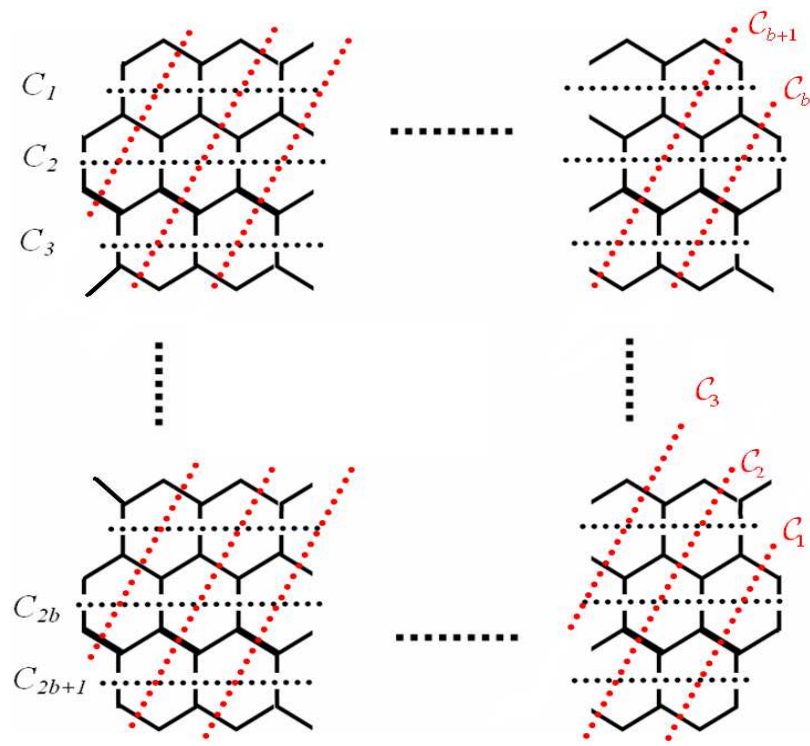

Figure. 2. The presentation of quasi-orthogonal cuts (qoc strips) of $B_{a, b}$ 
This implies that

$$
\begin{aligned}
S d\left(B_{a, b}, x\right) & =\sum_{c} m\left(B_{a, b}, c\right) \mathrm{x}^{\left|E\left(B_{a, b}\right)\right|-c} \\
& =\sum_{i=0}^{b} \mathrm{~m}\left(B_{a, b}, C_{2 i+1}\right) \mathrm{x}^{\left|E\left(B_{a, b}\right)\right|-\left|C_{2 i+1}\right|}+\sum_{i=1}^{b} \mathrm{~m}\left(B_{a, b}, C_{2 i}\right) \mathrm{x}^{\left|E\left(B_{a, b}\right)\right|-C_{2 i} \mid} \\
& +\sum_{i=1}^{b} \mathrm{~m}\left(B_{a, b}, \mathcal{C}_{i}\right) \mathrm{x}^{\left|E\left(B_{a, b}\right)\right|-\left|C_{i}\right|}+\mathrm{m}\left(B_{a, b}, \mathcal{C}_{b}\right) \mathrm{x}^{\left|E\left(B_{a, b}\right)\right|-\left|\mathcal{C}_{b}\right|} \\
& =(b+1) x^{\left|E\left(B_{a, b}\right)\right|-a}+b x^{\left|E\left(B_{a, b}\right)\right|-a-1} \\
& +4 \sum_{i=1}^{b} \mathrm{x}^{\left|E\left(B_{a, b}\right)\right|-2 i-1}+2(a-b-1) x^{\left|E\left(B_{a, b}\right)\right|-2 b-2}
\end{aligned}
$$

And also, from Table 2, $\forall a \leq b+1$ :

$$
\begin{aligned}
S d\left(B_{a, b}, x\right) & =\sum_{i=0}^{b} \mathrm{~m}\left(B_{a, b}, C_{2 i+1}\right) \mathrm{x}^{\left|E\left(B_{a, b}\right)\right|-\left|C_{2 i+1}\right|} \\
& +\sum_{i=1}^{b} \mathrm{~m}\left(B_{a, b}, C_{2 i}\right) \mathrm{x}^{\left|E\left(B_{a, b}\right)\right|-\left|C_{2 i}\right|}+\sum_{i=1}^{a} \mathrm{~m}\left(B_{a, b}, \mathcal{C}_{i}\right) \mathrm{x}^{\left|E\left(B_{a, b}\right)\right|-\left|\mathcal{C}_{i}\right|} \\
& =(b+1) x^{\left|E\left(B_{a, b}\right)\right|-a}+b x^{\left|E\left(B_{a, b}\right)\right|-a-1} \\
& +4 \sum_{i=1}^{a-1} \mathrm{x}^{\left|E\left(B_{a, b}\right)\right|-2 i-1}+2(b-a+1) x^{\left|E\left(B_{a, b}\right)\right|-2 a}
\end{aligned}
$$

Also, the Sadhana index of the hexagonal system is equal

where $\left|E\left(B_{a, b}\right)\right|=6 a b+5 a+b-4(=e)$.

$$
\begin{aligned}
S d\left(B_{a, b}\right) & =\left.S d^{\prime}\left(B_{a, b}, \mathrm{x}\right)\right|_{x=1} \\
& =\left.\frac{\partial\left((b+1) x^{\left|E\left(B_{a, b}\right)\right|-a}+b x^{\left|E\left(B_{a, b}\right)\right|-a-1}+4 \sum_{i=1}^{b} \mathrm{x}^{\left|E\left(B_{a, b}\right)\right|-2 i-1}+2(a-b-1) x^{\left|E\left(B_{a, b}\right)\right|-2 b-2}\right)}{\partial x}\right|_{x=1} \\
& =(e-a)(b+1)+b(e-a-1)+4 \sum_{i=1}^{b}(e-2 i-1)+2(a-b-1)(e-2 b-2) \\
& =(e(2 b+1)-2 a b-a-b)+\left(4 e b-4 b-8\left(\frac{b(b+1)}{2}\right)\right)+\left(2 e(a-b-1)+4 b-2 b^{2}-2 a b-2 a+2\right) \\
& =12 a^{2} b+24 a b^{2}+10 a^{2}+14 a b-2 b^{2}-16 a-14 b+6
\end{aligned}
$$

And also for $a \leq b+1$

$$
\begin{aligned}
S d\left(B_{a, b}\right) & =\left.\frac{\partial\left((b+1) x^{\left|E\left(B_{a, b}\right)\right|-a}+b x^{\left|E\left(B_{a, b}\right)\right|-a-1}+4 \sum_{i=1}^{a-1} \mathrm{x}^{\left|E\left(B_{a, b}\right)\right|-2 i-1}+2(b-a+1) x^{\left|E\left(B_{a, b}\right)\right|-2 a}\right)}{\partial x}\right|_{x=1} \\
& =(e-a)(b+1)+b(e-a-1)+4 \sum_{i=1}^{a-1}(e-2 i-1)+2(b-a+1)(e-2 a) \\
& =(e(2 b+1)-2 a b-a-b)+\left(4 e(a-1)-4 a+4-8\left(\frac{a(a-1)}{2}\right)\right)+\left(2 e(b-a+1)+4 a^{2}-4 a b-4 a\right) \\
& =12 a^{2} b+24 a b^{2}+10 a^{2}+10 a b+4 b^{2}-18 a-18 b+8
\end{aligned}
$$

Theorem 2: $\forall a \in \mathbb{N}$, the Sadhana polynomial of hexagonal system $B_{a, a}$ is equal to

\section{References}

$$
\begin{array}{r}
S d\left(B_{a, a}, x\right)=a x^{6 a^{2}+5 a-5}+(a+1) x^{6 a^{2}+5 a-4} \\
+4 \sum_{i=1}^{a-1} \mathrm{x}^{6 a^{2}+6 a-2 i-5}+2 x^{6 a^{2}+4 a-4}
\end{array}
$$

[1] N. Trinajstić, Chemical Graph Theory, CRC Press, Boca Raton, FL, (1992).

[2] H. Wiener, Structural determination of paraffin boiling points, J. Am. Chem. Soc. 69, 17-20. (1947).

where $\left|E\left(B_{a, b}\right)\right|=6 a^{2}+6 a-4$.

[3] R. Todeschini and V. Consonni, Handbook of Molecular Descriptors, Wiley-VCH, Weinheim, (2000).

Also, the Sadhana index of $B_{a, a}$ is equal to

$$
S d\left(B_{a, a}\right)=36 a^{3}+24 a^{2}-36 a+8
$$

[4] P.E. John, A.E. Vizitiu, S. Cigher, and M.V. Diudea, MATCH Commun. Math. Comput. Chem. 57, 479-484 (2007)

[5] M.V. Diudea, S. Cigher and P.E. John. MATCH Commun. 
Math. Comput. Chem, 60, 237-250 (2008).

[6] M.V. Diudea, S. Cigher, A.E. Vizitiu, O. Ursu and P. E. John, Croat. Chem. Acta, 79, 445 (2006).

[7] A.E. Vizitiu, S. Cigher, M.V. Diudea and M.S. Florescu, MATCH Commun. Math. Comput. Chem. 57, 457 (2007).

[8] M.V. Diudea, Hydrocarbons Using Orthogonal Cuts, J. Math. Chem (in print).

[9] M.V. Diudea, Omega Polynomial. Carpath. J. Math. 22 43-47 (2006).

[10] M.V. Diudea, S. Cigher, A.E. Vizitiu, M.S. Florescu and P.E. John, J. Math. Chem. 45 316-329 (2009).

[11] A.R. Ashrafi, M. Ghorbani and M. Jalali, Int. J. Chem. 47(A), 535 (2008).

[12] P.V. Khadikar, S. Joshi, A.V. Bajaj and D. Mandloi, Bioorg. Med.Chem. Lett. 14, 1187 (2004).

[13] P.V. Khadikar, V.K. Agrawal and S. Karmarkar, Bioorg. Med. Chem. 2, 10, 3499 (2002).

[14] A.R. Ashrafi, M. Ghorbani and M. Jalili. Computing Omega And Sadhana Polynomials of $C_{12 n+4}$ Fullerene. Digest. J. Nanomater. Bios. 4 (3), 403-406 (2009).

[15] M. Ghorbani. A Note on IPR Fullerene. Digest. J. Nanomater. Bios. 6 (2), 599-602 (2011).

[16] M. Ghorbani and M. Jalili. Omega And Sadhana Polynomials of Infinite Family of Fullerenes. Digest. J. Nanomater. Bios. 4 (1), 177 - 182 (2009).

[17] J. Yazdani and A. Bahrani. Padmakar-Ivan, Omega And Sadhana Polynomial of $H_{A} C_{5} C_{6} C_{7}$ Nanotubes. Digest. J. Nanomater. Bios. 4 (3), 507-510 (2009).

[18] A. Bahrani and J. Yazdani. Omega And Sadhana Polynomial of H-Naphtalenic Nanotubes And Nanotori. Digest. J. Nanomater. Bios. 3 (4), 309-314 (2008).

[19] M.R. Farahani, Omega and Sadhana Polynomials of Circumcoronene Series of Benzenoid. World Applied Sciences Journal. 20(9), 1248-1251, (2012).

[20] M.R. Farahani, K. Kato and M.P. Vlad. Omega Polynomials and Cluj-Ilmenau Index of Circumcoronene Series of Benzenoid. Studia Univ. Babes-Bolyai. 57(3) 177-182, (2012).

[21] S. Ling-Ling, W. Zhi-Ning and Z. Li-Qiang, Chinese Journal of Chemistry, 23(3), 245-250. (2005).

[22] Z. Bagheri, A. Mahmiani and O. Khormali. Iranian Journal of Mathematical Sciences and Informatics. 3(1), 31-39. (2008).

[23] M.R. Farahani, Omega Polynomial of a Benzenoid System, Submitted for publish, (2013).

[24] M.R. Farahani, Omega and related counting polynomials of Triangular Benzenoid $G_{n}$ and linear hexagonal chain $L H_{n}$. Journal of Chemica Acta. 2, 43-45. (2013).

[25] M.R. Farahani, Counting Polynomials of Benzenoid Systems. Romanian Academy Seri B. 15, (2013). In press.

[26] M.R. Farahani, Computing the Omega polynomial of an infinite family of the linear parallelogram $P(n, m)$. Journal of Advances in Chemistry. 1, (2013) In press.

[27] S. Klavzar. A Bird's Eye View of The Cut Method And A Survey of Its Applications In Chemical Graph Theory. MATCH Commun. Math. Comput. Chem. 60, 255-274 (2008).

[28] P.E. John, P.V. Khadikar and J. Singh. A method of computing the PI index of benzenoid hydrocarbons using orthogonal cuts. J. Math. Chem. 42(1), 27-45 (2007). 